\title{
PLASTICIDADE FENOTÍPICA E FRAÇÕES FOSFATADAS EM ESPÉCIES FLORESTAIS COMO RESPOSTA À APLICAÇÃO DE FÓSFORO ${ }^{1}$
}

Samuel Vasconcelos Valadares ${ }^{2}$, Leonardo Ferreira da Silva ${ }^{3}$, Rafael Vasconcelos Valadares ${ }^{4}$, Luiz Arnaldo Fernandes ${ }^{5}$, Júlio César Lima Neves ${ }^{6}$ e Regynaldo Arruda Sampaio ${ }^{7}$

\begin{abstract}
RESUMO - O experimento foi conduzido em casa de vegetação da Universidade Federal de Minas Gerais, com o objetivo de avaliar o crescimento inicial, a eficiência de uso do P e frações de fósforo em três espécies florestais submetidas a quatro níveis de $\mathrm{P}$. O delineamento experimental utilizado foi o inteiramente casualizado em arranjo fatorial ( 3 x 4) com três repetições. Mudas de gonçalo-alves (Astronium fraxinifolium Schott) e barbatimão (Stryphnodendron adstringens (Mart.) Coville), espécies nativas do Cerrado, e acácia-australiana (Acacia mangium Willd.), espécie exótica, foram cultivadas em Latossolo Vermelho-Amarelo adubado com $0,150,300$ e $600 \mathrm{mg} \mathrm{dm}^{-3} \mathrm{de}$ P. Aos 240 dias após a emergência, as plantas foram avaliadas quanto a altura, diâmetro do colo, produção de matéria seca, fósforo total solúvel em ácido e frações de P. As diferentes doses de fósforo influenciaram o crescimento inicial, a absorção e os teores totais e das frações de $\mathrm{P}$, nas três espécies. A espécie acácia-australiana apresentou maior crescimento em relação às espécies nativas nas maiores doses de $\mathrm{P}$. As espécies do Cerrado, por sua vez, apresentaram maior investimento em raízes e maior armazenamento de fósforo em formas inorgânicas, com destaque para o barbatimão. A relação P inorgânico:P orgânico mostrou-se adequada para a avaliação do status nutricional de $\mathrm{P}$ de espécies florestais.
\end{abstract}

Palavras-chave: Nutrição florestal; Crescimento; Espécies do Cerrado.

\section{PHENOTYPIC PLASTICITY AND PHOSPHATE FRACTIONS IN FOREST SPECIES AS A FUNCTION OF PHOSPHORUS APPLICATION}

\begin{abstract}
The experiment under greenhouse conditions was carried out at the Federal University of Minas Gerais, Brazil, aiming to evaluate the initial growth, efficiency of use of $P$ and phosphorus content in three forest species submitted to four levels of $P$. The experimental design was completely randomized in a factorial arrangement $3 \times 4$, with three replicates. Seedlings of Astronium fraxinifolium Schott and StryphnodendronAdstringens (Mart.) Coville, Cerrado native species, and Acacia mangium Willd., an exotic specie, were cultivated on a RedYellow Latosol treated with four phosphorous levels $\left(0,150,300\right.$ e $\left.600 \mathrm{mg} \mathrm{dm}^{-3}\right)$. After 240 days from the plants emergence the following variables were determined: height, root collar diameter, dry weight production, total phosphorous soluble in acid and P fractions. Different phosphorus doses influenced the initial growth, absorption, total content and content of $P$ fractions in the three studied species. Acacia mangium presented higher growth in the higher doses of phosphorus, in relation to the native species. The Cerrado species had higher enhancement of root and $P$ storage ability in inorganic forms, standing out Stryphnodendron adstringens. The relation inorganic $P$ :organic $P$ was considered as suitable for the $P$ nutritional status evaluation of forest species.
\end{abstract}

Keywords: Forest nutrition; Plant growth; Cerrado species.

\footnotetext{
${ }^{1}$ Recebido em 16.12.2011 aceito para publicação em 27.11.2014.

${ }^{2}$ Universidade Federal de Viçosa, Departamento de Solos, Viçosa, MG - Brasil. E-mail: <samuel.valadares@ufv.br>.

${ }^{3}$ Universidade Estadual de Santa Cruz, Centro de Biotecnologia e Genética, Ilheus, BA - Brasil. E-mail: <leonardoufmg3@yahoo.com.br>. ${ }^{4}$ Universidade Federal de Minas Gerais, Instituto de Ciências Agrárias, Montes Claros, MG- Brasil. E-mail: <rafaelvvaladares@hotmail.com>. ${ }^{5}$ Universidade Federal de Minas Gerais, UFMG, Instituto de Ciências Agrárias, Montes Claros, MG- Brasil. E-mail: <larnaldo@ufmg.br> . ${ }^{6}$ Universidade Federal de Viçosa, Departamento de Solos, Viçosa, MG - Brasil. E-mail: <julio_n2003@yahoo.com.br>.

${ }^{7}$ Universidade Federal de Minas Gerais, Instituto de Ciências Agrárias - Montes Claros, MG - Brasil. E-mail: <rsampaio@ufmg.br>.
} 


\section{INTRODUÇ̃̃O}

Para o melhor entendimento e exploração das interações solo-planta, a avaliação do comportamento nutricional das espécies é fundamental.

São muito adequadas pesquisas que visam ao estudo de genótipos mais eficientes em se desenvolver sob condições diversas de ambiente. Esses estudos adquirem grande importância nas condições de solos tropicais cujas limitações, quanto à disponibilidade de nutrientes, estão entre os principais fatores que determinam a produtividade dos cultivos agrícolas e florestais (BARROS; COMERFORD, 2002), especialmente no bioma Cerrado, o segundo maior bioma brasileiro (KLINK; MACHADO, 2005).

Associados à eficiência nutricional, fatores socioeconômicos devem ser considerados na escolha de espécies florestais a serem utilizadas em programas de reflorestamento. Assim, trabalhos que visam ao estudo nutricional de espécies de interesse para as populações rurais, nativas do bioma Cerrado, associados a programas de manejo participativo para sua utilização, são necessários e têm sido bastante incentivados pelas instituições de fomento à pesquisa no Brasil.

Informações sobre os requerimentos nutricionais, bem como sobre a capacidade de adaptação de espécies florestais nativas do bioma Cerrado, sua plasticidade fenotípica, são escassas, principalmente no que se refere ao fornecimento e à eficiência de utilização de nutrientes, como o fósforo (RESENDE et al., 1999; SCHUMACHER et al., 2004), um dos nutrientes mais limitantes da produção vegetal em solos tropicais (NOVAIS; SMYTH, 1999; SANTOS et al., 2008).

O acúmulo de fósforo na planta está relacionado às variações de sua disponibilidade no solo, principalmente em sua forma inorgânica (Pi vacuolar), que é acumulada no vegetal com função de reserva e não possui função metabólica imediata (BIELESKI, 1973). Quando a quantidade de P absorvida é menor que a demanda pela célula, o Pi do vacúolo retorna ao citoplasma, atendendo às exigências metabólicas celulares (BIELESKI; FERGUSON, 1983; SANTOS et al., 2008). Por isso, a resposta de espécies distintas à fertilização fosfatada, bem como sua adaptabilidade a condições restritivas quanto a esse nutriente, pode ser explicada pela dinâmica das frações de P na planta (FERNANDES et al., 2000). Desse modo, o acompanhamento dos teores de P inorgânico (Pi) e orgânico $(\mathrm{Po})$ pode auxiliar no entendimento do comportamento em crescimento, bem como na eficiência de utilização desse nutriente pelas plantas (FERNANDES et al., 2000), permitindo melhor compreensão da relação solo-planta sob condições diversas que envolvem a disponibilidade desse nutriente.

Objetivou-se, com este estudo, avaliar as modificações no crescimento e na distribuição das frações fosfatadas nas folhas de duas espécies florestais nativas do Cerrado e uma exótica, utilizada para fins comparativos, sob diferentes disponibilidades de P no solo.

\section{MATERIAL E MÉTODOS}

O experimento foi realizado no Instituto de Ciências Agrárias da Universidade Federal de Minas Gerais, na cidade de Montes Claros, MG, durante o período de março a dezembro de 2007. Os tratamentos consistiram de três espécies florestais e quatro doses de fósforo em arranjo fatorial $(3 \times 4)$, no delineamento inteiramente casualizado com três repetições.

As espécies estudadas foram Astronium fraxinifoliumm, Stryphnodendron adstringens e Acacia mangium, todas três classificadas como pioneiras (ARCO-VERDE, 2002; LORENZI, 2002). As duas primeiras, nativas do Cerrado brasileiro, são denominadas gonçalo-alves e barbatimão, respectivamente, e utilizadas para fins medicinais na região Norte de Minas Gerais. A terceira, chamada de acácia-australiana, é espécie exótica muito empregada na recuperação de áreas degradadas.

Para obter um gradiente da disponibilidade de fósforo, foram aplicadas as doses de 0, 150, $300 \mathrm{e}$ $600 \mathrm{mg} \mathrm{dm}^{-3} \mathrm{de}$ P.

Cada unidade experimental foi constituída por um vaso de três $\mathrm{dm}^{3}$ preenchido por Latossolo VermelhoAmarelo distrófico, coletado na camada de 0 a $20 \mathrm{~cm}$ de profundidade, com os seguintes atributos físicos e químicos, determinados conforme Embrapa (1997): $\mathrm{pH}$ em água $=4,9$; $\mathrm{P}$ Mehlich- $1=0,8 \mathrm{mg} \mathrm{dm}^{-3} ; \mathrm{K}=$ 2,0 mmolc dm ${ }^{-3} ; \mathrm{Ca}=12,0$ mmolc dm $^{-3} ; \mathrm{Mg}=5,0$ mmolc $\mathrm{dm}^{-3} ; \mathrm{Al}=16,0$ mmolc dm ${ }^{-3} ; \mathrm{H}+\mathrm{Al}=191,5$ mmolc dm$^{-}$ 3; CTC efetiva $=35,1 \mathrm{mmolc} \mathrm{dm}^{-3}$; CTC potencial $=$ 210,6 mmolc dm ${ }^{-3}$; saturação por $\mathrm{Al}=46,0 \%$; saturação por bases $=9,0 \%$; matéria orgânica do solo $=15,5$

Revista Árvore, Viçosa-MG, v.39, n.2, p.225-232, 2015 
$\mathrm{g} \mathrm{kg}^{-1}$; areia $=320 \mathrm{~g} \mathrm{~kg}^{-1}$; silte $=440 \mathrm{~g} \mathrm{~kg}^{-1}$; e argila $=240 \mathrm{~g} \mathrm{~kg}^{-1}$.

Trinta dias antes do plantio, efetuou-se a aplicação de $\mathrm{CaCO}_{3}$, de forma a elevar o $\mathrm{pH}$ do solo a 5,5 e 15 dias antes da semeadura, uma adubação básica com $30 \mathrm{mg}$ de $\mathrm{N}, 100 \mathrm{mg}$ de $\mathrm{K}, 50 \mathrm{mg}$ de $\mathrm{S}, 0,5 \mathrm{mg}$ de B, 1,5 $\mathrm{mg}$ de $\mathrm{Cu}, 5,0 \mathrm{mg}$ de $\mathrm{Zn}$ e 1,5 $\mathrm{mg}$ de Fe por $\mathrm{dm}^{3}$ de solo, aplicados na forma de sais p.a.

Em cada vaso foram semeadas seis sementes e realizado o desbaste 20 dias após a emergência, selecionando-se duas plântulas uniformes por parcela. A umidade do solo foi mantida próxima à capacidade de campo, por meio de pesagens diárias dos vasos e reposição da água evapotranspirada.

Após 240 dias da emergência das plantas, foram avaliados o diâmetro do caule na altura do colo, altura das plantas e coletados aproximadamente $500 \mathrm{mg}$ de folhas por parcela, para determinação das frações fosfatadas (o tecido foliar foi coletado na primeira folha expandida a partir do ápice da planta).

A plasticidade fenotípica das espécies foi avaliada pelo comportamento das seguintes variáveis, em resposta a diferentes níveis de $\mathrm{P}$ no solo: matéria seca de parte aérea (MSPA), matéria seca de raiz (MSRA), fósforo total na folha e relação MSRA/MSPA.

A metodologia do fracionamento de P utilizada foi a proposta por Hogue et al. (1970) e Smillie e Krotkov (1960), modificada por Martinez (1992).

A eficiência de uso de fósforo foi calculada pela equação $\mathrm{MST} / \mathrm{Pts}_{2}$ em que MST = matéria seca total e Pts = teor de fósforo total solúvel em ácido.

Para a análise estatística, utilizou-se o software SAEG 9.1. Os dados foram submetidos à análise de variância. A resposta das espécies estudadas à adição de $\mathrm{P}$ foi avaliada por meio da análise de regressão $(p<0,05$ pelo teste $\mathrm{t}$ ).

\section{RESULTADOS}

A acácia-australiana respondeu à adição de $\mathrm{P}$ e apresentou maiores incrementos nos valores de altura, diâmetro do colo e matéria seca da parte aérea em relação às espécies nativas do Cerrado (Tabela 1). O diâmetro do colo e a MSPA aumentaram linearmente com o incremento das doses de $\mathrm{P}$, e as variáveis MSRA e altura tiveram incremento quadrático. A altura máxima
(23,67 cm) e a máxima MSRA $\left(3,36 \mathrm{~g} \mathrm{vaso}^{-1}\right)$ foram obtidas nas doses correspondentes a 522 e $550 \mathrm{mg} \mathrm{dm}^{-3}$, respectivamente.

Para a espécie gonçalo-alves, o aumento da adubação fosfatada resultou em resposta quadrática positiva para altura, diâmetro do colo, MSPA e MSRA(Tabela 1). Os máximos crescimentos em altura $(8,64 \mathrm{~cm})$ e em diâmetro do colo $(2,55 \mathrm{~mm})$ foram obtidos nas doses correspondentes a 328 e $278 \mathrm{mg} \mathrm{dm}^{-3} \mathrm{de} \mathrm{P}$, respectivamente. As máximas produções de $\operatorname{MSPA}\left(0,63 \mathrm{~g} \mathrm{vaso}^{-1}\right)$ e de MSRA $\left(3,23 \mathrm{~g} \mathrm{vaso}^{-1}\right)$ foram observadas nas doses correspondentes a 300 e $295 \mathrm{mg} \mathrm{dm}^{-3} \mathrm{de}$ P, respectivamente.

Os níveis de $\mathrm{P}$ estudados não afetaram o crescimento em altura e diâmetro do colo do barbatimão. No entanto, as produções de MSPA e MSRA aumentaram linearmente com o incremento das doses de P. Ressalta-se, ainda, que a magnitude da resposta dessa espécie no que se refere aos incrementos na MSPA foi inferior às demais. Em contrapartida, essa mesma espécie apresentou maiores produções de matéria seca de raiz em relação às demais (Tabela 1 ).

A relação MSRA/MSPA foi marcadamente superior nas espécies nativas em comparação com a espécie exótica (Figura 1). Isso é particularmente destacado no caso do barbatimão, para o qual o aumento da disponibilidade de fósforo resultou em resposta quadrática positiva nessa relação, o que não ocorreu com as outras duas espécies. A relação MSRA/MSPA não foi significativamente alterada no caso da espécie gonçalo-alves, contrariamente ao ocorrido com a acáciaaustraliana, que demonstrou plasticidade com relação a essa variável. Na dose zero, essa espécie apresentou baixa relação MSRA/MSPA, com grande incremento inicial, seguido de decréscimo com o incremento das doses de P.

Diferenças no teor foliar de $\mathrm{P}$ total solúvel em ácido (Pts) e nas frações de P nas folhas ocorreram entre as três espécies $(P<0,05)$.

Na avaliação do Pts e P orgânico solúvel em ácido (Po), observaram-se maiores teores nas espécies nativas em relação à espécie exótica até a dose de $150 \mathrm{mg} \mathrm{dm}^{-3} \mathrm{de}$ $\mathrm{P}$, porém, sob maiores disponibilidades do nutriente, a acácia apresentou maiores teores de Pts e Po. De modo geral, com o aumento das doses de P, a acáciaaustraliana apresentou os maiores valores de Pts e Po e menores de $\mathrm{Pi}$, em comparação com as espécies nativas do Cerrado (Tabela 2).

Revista Árvore, Viçosa-MG, v.39, n.2, p.225-232, 2015 
Tabela 1 - Equações de regressão referentes a altura, matéria seca de raiz (MSRA), diâmetro do colo e matéria seca da parte aérea (MSPA), em função da aplicação de P nas três espécies florestais.

Table 1 -Regression equations for plant height, root dry weight (RDW), root collar diameter, and shoot dry weight (SDW), as a function of $P$ application in three forest species.

\begin{tabular}{|c|c|c|c|c|c|}
\hline Espécie & Variáveis & Equações & $\mathrm{R}^{2}$ & $\mathrm{x} \mathrm{p} / \hat{\mathrm{y}} \max \left(\mathrm{mgdm}^{-3}\right)$ & Ganho $(\%)$ \\
\hline \multirow[t]{4}{*}{ Gonçalo-alves } & Altura $(\mathrm{cm})$ & $\hat{\mathrm{y}}=3,866+0,032 * * \mathrm{x}-0,00005 * * \mathrm{x}^{2}$ & 0,98 & 320 & 131 \\
\hline & $\operatorname{MSRA}(\mathrm{g})$ & $\hat{\mathrm{y}}=0,111+0,017 * * \mathrm{x}-0,00003 * * \mathrm{x}^{2}$ & 0,97 & 278 & 195 \\
\hline & Diâmetro $(\mathrm{mm})$ & $\hat{\mathrm{y}}=0,789+0,011 * * \mathrm{x}-0,00002 * * \mathrm{x}^{2}$ & 0,99 & 250 & 1171 \\
\hline & $\operatorname{MSPA}(\mathrm{g})$ & $\hat{\mathrm{y}}=0,032+0,003 * \mathrm{x}-0,000006 * \mathrm{x}^{2}$ & 0,99 & 283 & 2170 \\
\hline \multirow[t]{4}{*}{ Barbatimão } & Altura $(\mathrm{cm})$ & $\hat{\mathrm{y}}=2,318$ & & 0 & 0 \\
\hline & MSRA (g) & $\hat{\mathrm{y}}=0,344-0,002 * * \mathrm{x}$ & 0,92 & 0 & 0 \\
\hline & Diâmetro $(\mathrm{mm})$ & $\hat{y}=1,378$ & & 600 & 91 \\
\hline & $\operatorname{MSPA}(\mathrm{g})$ & $\hat{\mathrm{y}}=0,132-0,0002 * \mathrm{x}$ & 0,68 & 413 & 75 \\
\hline \multirow[t]{4}{*}{ Acácia } & Altura $(\mathrm{cm})$ & $\hat{y}=7,147+0,063 * * x-0,00006^{* *} x^{2}$ & 0,98 & 563 & 183 \\
\hline & $\operatorname{MSRA}(\mathrm{g})$ & $\hat{\mathrm{y}}=0,336+0,011 * * \mathrm{x}-0,00001 * * \mathrm{x}^{2}$ & 0,99 & 600 & 3552 \\
\hline & Diâmetro $(\mathrm{mm})$ & $\hat{\mathrm{y}}=0,718+0,042 * * \mathrm{x}$ & 0,93 & 600 & 4247 \\
\hline & $\operatorname{MSPA}(\mathrm{g})$ & $\hat{\mathrm{y}}=0,678+0,004 * * \mathrm{x}$ & 0,99 & 428 & 890 \\
\hline
\end{tabular}

**Significativo pelo teste $\mathrm{t}(P=0,01) \mathrm{e}$ *significativo pelo teste $\mathrm{t}(P=0,05)$.

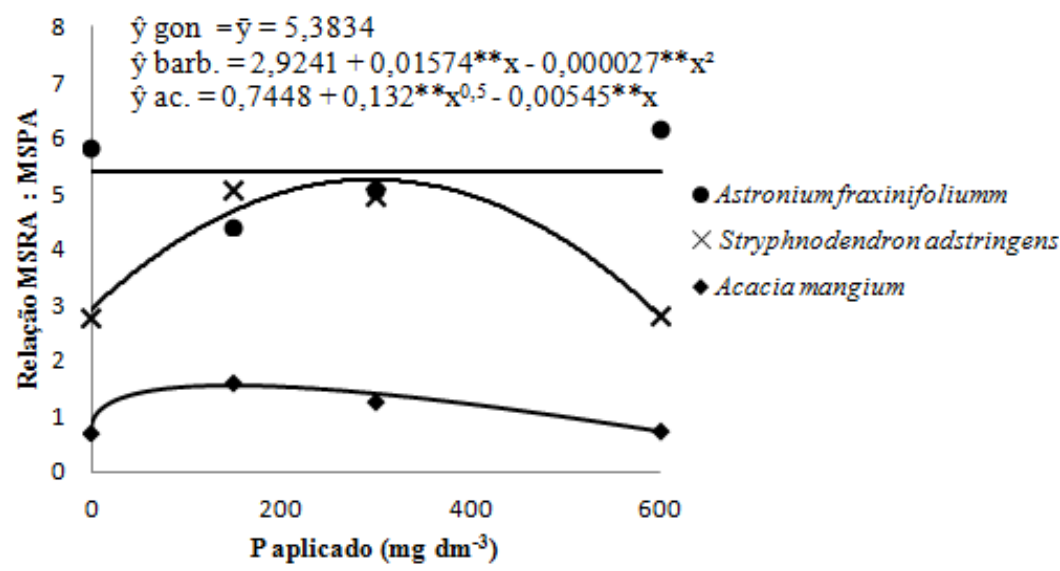

Figura 1 - Efeito da adubação com P na relação raiz:parte aérea de espécies florestais nativas do Cerrado e uma espécie florestal exótica. $* *$ Significativo pelo teste $\mathrm{t}(P=0,01)$ e $*$ significativo pelo teste $\mathrm{t}(P=0,05)$.

Figure 1 - Effect of $P$ fertilization on root:shoot ratios of forest native species of Cerrado and an exotic forest specie. **Significant by the test $(P=0.01)$ and *significant by the test $(P=0.05)$.

Diferentes padrões foram observados na avaliação do relacionamento entre o teor total de fósforo solúvel em ácido, fração Pi e fração Po, bem como da relação Pi:Po com o crescimento das três espécies (Figuras 1 e 2). Maior crescimento da espécie acácia-australiana foi observado com o aumento da relação Pi:Po, havendo tendência à estabilização quando essa relação foi superior a 1. Relacionamento quadrático foi verificado entre as variáveis Pi:Po e MST na espécie gonçaloalves. O crescimento máximo dessa espécie foi obtido quando a relação Pi:Po foi igual a 1,45. A variável
MST não se relacionou aos valores de Pi:Po para o barbatimão.

\section{DISCUSSÃO}

São distintas as respostas das três espécies estudadas ao aumento da disponibilidade de fósforo, com destacada diferença entre a espécie exótica e as espécies nativas do Cerrado (Tabela 1). Essas constatações retratam os diferentes padrões de alocação de carbono entre essas espécies e se adequam às observações de Resende et al. (1999), que, assim como

Revista Árvore, Viçosa-MG, v.39, n.2, p.225-232, 2015 
Tabela 2 - Fósforo total solúvel em ácido, orgânico e inorgânico, em três espécies florestais, sob diferentes níveis de aplicação do nutriente.

Table 2 - Total acid-soluble P, organic and inorganic phosphorus in three forest species under different nutrient application levels.

\begin{tabular}{|c|c|c|c|}
\hline Espécie & Variáveis & Equações & $\mathrm{R}^{2}$ \\
\hline Gonçalo-alves & $\begin{array}{l}\text { Fósforo total } \\
\text { Fósforo orgânico } \\
\text { Fósforo inorgânico }\end{array}$ & $\begin{array}{c}\hat{y}=0,476+0,002 * * x-0,000003 * * x^{2} \\
\hat{y}=636,4-1,043 * * x \\
\hat{y}=297,2+0,727 * * x-0,0003 * x^{2}\end{array}$ & $\begin{array}{l}0,96 \\
0,98 \\
0,99\end{array}$ \\
\hline Barbatimão & $\begin{array}{l}\text { Fósforo total } \\
\text { Fósforo orgânico } \\
\text { Fósforo inorgânico }\end{array}$ & $\begin{array}{c}\hat{y}=0,756 \\
\hat{y}=180,2+3,637 * x-0,006 * * x^{2} \\
\hat{y}=113,2+1,234 * x\end{array}$ & $\begin{array}{l}0,98 \\
0,98\end{array}$ \\
\hline Acácia & $\begin{array}{l}\text { Fósforo total } \\
\text { Fósforo orgânico } \\
\text { Fósforo inorgânico }\end{array}$ & $\begin{array}{l}\hat{y}=0,552+0,001 * *_{x} \\
\hat{y}=444,2+1,064 * *_{x} \\
\hat{y}=152,6+0,826 * * x\end{array}$ & $\begin{array}{l}0,93 \\
0,86 \\
0,91\end{array}$ \\
\hline
\end{tabular}

**Significativo pelo teste $\mathrm{t}(P=0,01)$ e *significativo pelo teste $\mathrm{t}(P=0,05)$.

neste estudo, obtiveram menor resposta à fertilização por espécies de crescimento mais lento, como as espécies nativas estudadas.

Embora as três espécies pertençam a um mesmo grupo sucessional, o maior crescimento da espécie exótica em relação às nativas, quanto aos valores de altura, diâmetro do colo e produção de matéria seca da parte aérea, em diferentes níveis de fósforo, pode ser atribuído, em parte, às diferenças de plasticidade fenotípica dessa espécie em relação às demais, quanto à alocação de carbono para a parte aérea, à medida que aumenta a disponibilidade de P.

Em condições de alta disponibilidade de $\mathrm{P}$, a relação MSRA/MSPA da acácia-australiana diminui (Figura 1). Associa-se a tal fato o maior desenvolvimento de órgãos fotossinteticamente ativos, condição necessária para que o ritmo de crescimento dessa espécie seja mais intenso, comparativamente às demais ( $\mathrm{LYNCH}$; BROWN, 2001; HAMMOND et al., 2004).

Os resultados estão de acordo com os estudos de Daniel et al. (1997), que reportaram o maior desenvolvimento de parte aérea em relação ao sistema radicular de mudas de acácia-australiana em doses maiores que $400 \mathrm{mg} \mathrm{dm}^{-3}$ de P. Essa característica, associada à morfologia de seu sistema radicular, torna essa espécie muito eficiente na utilização do $\mathrm{P}$, o que corrobora os estudos de Nguyen et al. (2006), que destacaram também em seus estudos a Acacia mangium,_por sua elevada eficiência de utilização de P.

De modo diferente em relação à espécie exótica, a morfologia das espécies nativas do Cerrado evidencia as raízes como drenos preferenciais de fotoassimilados, mesmo com o aumento da disponibilidade de P (Figura 1), indicando maior adaptação à baixa disponibilidade de nutrientes no solo e a condições de déficit hídrico, tal qual normalmente ocorre no bioma Cerrado. Assim, sob o enfoque deste estudo, essas espécies são recomendadas para programas de reflorestamento e de recuperação de áreas degradadas, para condições mais restritivas de solo e clima.

É notável também que as duas espécies do Cerrado apresentem padrões diferenciados de resposta ao aumento da disponibilidade de $\mathrm{P}$, vez que gonçalo-alves apresenta maior desenvolvimento de parte aérea, em comparação com o barbatimão, em resposta ao aumento da quantidade de P aplicada (Figura 1 e Tabela 1).

Torna-se, portanto, evidente a necessidade de serem consideradas as diferenças de comportamento entre as espécies nativas do Cerrado, para melhor exploração da variabilidade entre elas e sua interação com as diferentes condições de solo. O que está de acordo com outros estudos, posto ser o comportamento diferencial das espécies quanto à nutrição geneticamente controlado (BASU et al., 2011).

De modo similar às observações encontradas neste estudo sobre gonçalo-alves, Gomes et al. (2004) constataram respostas positivas à adubação fosfatada de angico-branco. Da mesma forma, Schumacher et al. (2004), trabalhando com adubação fosfatada de mudas de angico-vermelho, encontraram resposta quadrática positiva para todas as variáveis estudadas. Também se alinham aos resultados deste estudo as observações de Santos et al. (2008), que verificaram padrões variados

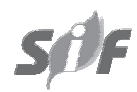

Revista Árvore, Viçosa-MG, v.39, n.2, p.225-232, 2015 
de resposta de algumas espécies florestais nativas do Cerrado à fertilização com $\mathrm{P}$.

Os padrões de absorção de fósforo são diferenciados entre as três espécies, bem como de partição do $\mathrm{P}$ absorvido.

Embora os teores totais de $\mathrm{P}$ aumentem em todas as espécies com o incremento das doses desse nutriente, a magnitude dos incrementos torna acentuadas as diferenças fenotípicas entre os materiais estudados,
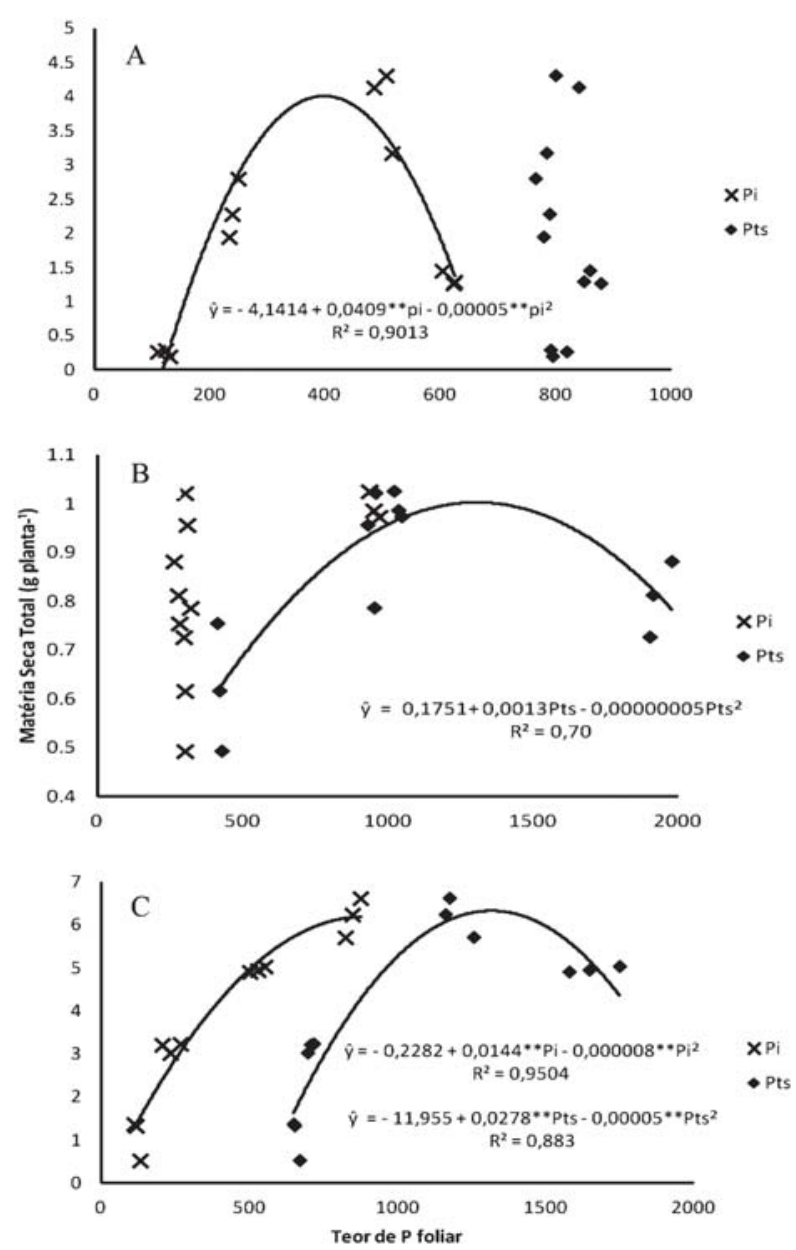

Figura 2 - Relações entre massa seca total (MST) e P total solúvel (Pts) em ácido e $\mathrm{P}$ inorgânico (Pi) em Astronium fraxinifolium (A), Stryphnodendron adstringens (B) e Acacia mangium (C), durante seu desenvolvimento inicial.

Figure 2 - Relations between total dry weight (TDW)_and phosphorus total soluble (Pts) and $P$ inorganic $(P i)$ in Astronium fraxinifolium (A), Stryphnodendron adstringens (B) and Acacia mangium (C), during the initial development. sendo as diferenças ainda maiores nas frações de P. De forma semelhante às observações deste estudo, Santos et al. (2006), trabalhando com frações de fósforo em gramíneas forrageiras, constataram aumento nos teores totais de $\mathrm{P}$ e que os teores de Pi aumentaram diretamente com as doses de fósforo aplicado ao solo.

Plantas adaptadas a solos de baixa fertilidade apresentam, em geral, altas concentrações de fósforo e de outros nutrientes na parte aérea. Isso está normalmente associado à menor suscetibilidade dessas espécies a condições de estresse nutricional (OSTERTAG, 2010). Essas espécies não se caracterizam por alta eficiência de absorção, sendo os elevados teores de nutrientes na biomassa função de suas lentas taxas de crescimento (FERNANDES et al., 2000). Portanto, os maiores teores de Pi no barbatimão, nas condições de baixo suprimento de $\mathrm{P}$, indicam grande adaptação dessa espécie às condições de baixa disponibilidade de nutrientes, seguida de gonçalo-alves e acácia-australiana.

Propõe-se, em contraposição com a maior resposta em crescimento inicial da espécie exótica, que o barbatimão suporta maior déficit de $\mathrm{P}$ que a acáciaaustraliana e a espécie gonçalo-alves estaria intermediária entre ambas.

Essas constatações são reforçadas pelo relacionamento entre o teor de Pts e a fração $\mathrm{Pi}$, bem como a relação Pi:Po e a produção de matéria seca total das espécies (Figuras 1 e Figura 2). Os resultados sugerem ainda que a diferenciação das frações metabólicas e não metabólicas do $\mathrm{P}$ na planta é conveniente para o entendimento do comportamento diferencial das plantas em crescimento (FERNANDES et al.,2000; MARTINEZ et al.,2005 e SANTOS et al., 2008;).

\section{CONCLUSÕES}

As espécies Astronium fraxinifolium e Stryphnodendron adstringens, nativas do Cerrado, são responsivas à fertilização com fósforo durante seu crescimento inicial.

Os padrões de resposta da partição de biomassa na planta e das frações de fósforo solúvel em ácido em função da adição de P são diferenciados entre as espécies estudadas.

A relação Pi:Po pode ser utilizada na predição de resposta à adubação com $\mathrm{P}$ por algumas espécies florestais.

Revista Árvore, Viçosa-MG, v.39, n.2, p.225-232, 2015 


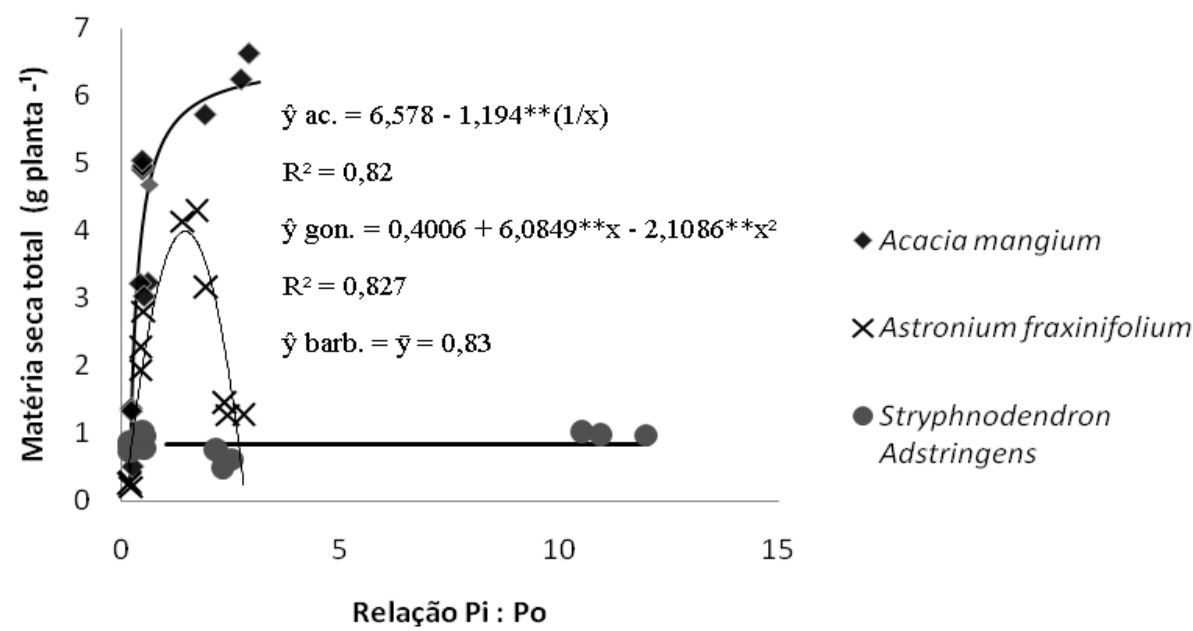

Figura 3 - Matéria seca total como função da relação fósforo inorgânico solúvel em ácido e fósforo orgânico (Pi:Po), em três espécies florestais.

Figure 3 - Total dry weight as a function of the relation between soluble inorganic phosphorus in acid and organic P phosphorus (Pi:Po) in three forest species.

\section{REFERÊNCIAS}

ARCO-VERDE, M.F. Potencialidades e usos da Acacia mangium Willd. no estado de Roraima. Boa Vista: Embrapa Roraima, 2002. 18p. (Documentos, 6).

BARROS, N.F.; COMERFORD, N.B.

Sustentabilidade da produção de florestas plantadas na região tropical. In: ALVAREZ V., V.H.; SCHAFER, C.E.G.R.; BARROS, N.F.; MELLO, J.W.V.; COSTA, L.M. (Ed.). Tópicos em ciência do solo. Viçosa, MG: Sociedade Brasileira de Ciência do Solo, 2002. v.2. p.487-592.

BASU, A.; BROWN, K.M.; PAL, A. Detailed quantitative analysis of architectural traits of basal roots of young seedlings of Phaseolus vulgaris L. in response to auxin and ethylene. Plant Physiology Preview, v.155, n.4, p.2056-65, 2011.

BIELESKI, R.L. Phosphate pools, phosphate transport and phosphate availability. Annual Review of Plant Physiology, v.24, p.225-252, 1973.

BIELESKI, R.L.; FERGUSON, J.B. Physiology and metabolism of phosphate and its compounds. In: PIRSON, A.; ZIMMERMANN, M.H. (Ed.).

Encyclopedia of plant physiology: inorganic plant nutrition. Berlin: Springer, 1983. p.442-449.
DANIEL, O.; VITORINO, A.C.T.; ALOVISI, A.A.; MAZZOCHIN, L.; TOKURA, A.M.; PINHEIRO, E.R.P.; SOUZA, E.F. Aplicação de fósforo em mudas de Acacia mangium Willd. Revista Árvore, v.21, n.2, p.163-168, 1997.

LYNCH, J.P.; BROWN, K.M. Topsoil foraging an architectural adaptation of plants to low phosphorus availability. Plant and Soil, v.23, p.225-237, 2001.

EMPRESA BRASILEIRA DE PESQUISA AGROPECUÁRIA - EMBRAPA. Serviço Nacional de Levantamento e Conservação de Solo. Manual de métodos de análise de solo. Rio de Janeiro: 1997. 212p.

FERNANDES, L.A; FURTINI NETO, A.E.; FONSECA, F.C.; VALE, F.R. Crescimento inicial, níveis críticos de fósforo e frações fosfatadas em espécies florestais. Pesquisa Agropecuária Brasileira, v.35, n.6, p.1191-1198, 2000.

GOMES, K. C. O.; PAIVA, H. N.; NEVES, J. V. L.; BARROS, N. F.; SILVA, S. R. Influência da saturação por bases e do fósforo no crescimento de mudas de angico-branco. Revista Árvore, v.28, n.6, p.785-792, 2004.

HAMMOND, J.P.; BROADLEY, M.R.; WHITE, P.J. Genetic responses to phosphorus

Revista Árvore, Viçosa-MG, v.39, n.2, p.225-232, 2015 
deficiency. Annals of Botany, v.94, n.3, p.323$332,2004$.

HOGUE, E.; WILCOX, G.E.; CANTLIFFE, D.J. Effect of soil phosphorus levels on phosphate fractions in tomato leaves. American Society for Horticultural Science Journal, v.95, n.1, p.174-176, 1970.

KLINK, A.C.; MACHADO, R.B. A conservação do Cerrado brasileiro. Megadiversidade, v.1., n.1, p.147-155, 2005.

LORENZI, H. Árvores brasileiras: manual de identificação e cultivo de plantas arbóreas do Brasil. Nova Odessa: Instituto Plantarum, 2002. 384p.

MARTINEZ, H.E.P. Cinética da absorção e das frações fosfatadas em sete variedades de soja (Glycine max, L. Merril). Viçosa, MG: Universidade Federal de Viçosa, 1992. (Relatório de Pós-Doutoramento)

MARTINEZ, H.E.P.; NOVAIS, R.F.; RODRIGUES, L.A.; SACRAMENTO, L.V.S. Phosphate forms in plant and their internal buffering in five soybean cultivars. Revista Brasileira de Ciência do Solo, v.29, n.2, p.249-257, 2005.

NGUYEN, N.T.; MOHAPATRA, P.K.; FUJITA, K. Elevated $\mathrm{CO}_{2}$ alleviates the effects of low $\mathrm{P}$ on the growth of $\mathrm{N}_{2}$ fixing Acacia auricuriformis and Acacia mangium. Plant and Soil, v.285, p.369-379, 2006.

NOVAIS, R.F.; SMYTH, T.J. Fósforo em solo e planta em condições tropicais. Viçosa,
MG: Universidade Federal de Viçosa - DPS, 1999. 399p.

OSTERTAG, R. Foliar nitrogen and phosphorus accumulation responses after fertilization: an example from nutrientlimited Hawaiian forests. Plant and Soil, v.334, n.1, p.85-98, 2010.

RESENDE, A.V.; FURTINI NETO, A.E.; MUNIZ, J.A.; CURI, N.; FAQUIN, V. Crescimento inicial de diferentes espécies florestais de diferentes grupos sucessionais em resposta a doses de fósforo. Pesquisa Agropecuária Brasileira, v.34, n.11, p.2071-2081, 1999.

SANTOS, I. P. A.; PINTO, J. C.; FURTINI NETO, A. E.; MORAIS, A. R.; MESQUITA. E. E.; FARIA. D. J. G.; ROCHA. G. P. Frações de fósforo em gramíneas forrageiras tropicais sob fontes e doses de fósforo. Ciência e Agrotecnologiaca, v.30, n.5, p.961-970, 2006.

SANTOS, J.Z.L.; RESENDE, A.V.; FURTINI NETO, A.E.; CORTE, E.F. Crescimento, acúmulo de fósforo e frações fosfatadas em mudas de sete espécies arbóreas nativa Revista Árvore, v.32, n.5, p.799-807, 2008.

SCHUMACHER, M.V.; CECONI, D.E.; SANTANA, C.A. Influência de diferentes doses de fósforo no crescimento de mudas de angico vermelho (Parapiptadenia rigida Bentham Brenan). Revista Árvore, v.28, n.1, p.149155, 2004.

SMILLIE, R.M.; KROTKOV, G. The estimation of nucleic acids in some algae and higher plants. Canadian Journal of Botany, v. 38 , n.1, p.31-49, 1960. 